\title{
Inhibition of p21 activated kinase enhances tumour immune response and sensitizes pancreatic cancer to gemcitabine
}

\author{
KAI WANG, NHI HUYNH, XIAO WANG, GRAHAM BALDWIN, MEHRDAD NIKFARJAM and HONG HE \\ Department of Surgery, University of Melbourne, Austin Health, Heidelberg, Victoria 3048, Australia
}

Received August 11, 2017; Accepted October 18, 2017

DOI: 10.3892/ijo.2017.4193

\begin{abstract}
Pancreatic ductal adenocarcinoma (PDA) is one of the major types of cancer that exhibit high mortality worldwide because of the late diagnosis and the lack of effective treatment. Immunotherapy appears to be ineffective in PDA treatment due to the existence of a unique immune-suppressive microenvironment in PDA. Gemcitabine-based therapy is still the most commonly used chemotherapy to treat PDA patients with only marginal increased survival rates. This prompted us to continue the search for more effective therapy for PDA treatment. The effects of p21 activated kinases (PAKs) on tumour immune response and gemcitabine response were examined in PDA. An orthotopic murine PDA model, in which pancreatic cancer cells were injected to the tail of pancreas, was used. The mice were treated with PAK inhibitor, PF-3758309, plus or minus gemcitabine. Tumour growth was measured by volume and weight. Tumour immune response was determined by flow cytometry analysis of splenic cells and immunohistochemical staining of intratumoural lymphocytes. Inhibition of PAKs by PF-3758309, not only suppressed tumour growth, but also stimulated tumour immune response by increasing the numbers of splenic and intratumoural T lymphocytes. Furthermore, inhibition of PAKs decreased PDA cell growth synergistically with gemcitabine in vitro and in vivo. The dual effects of inhibition of PAKs make PAK-targeted therapy more potent for the treatment of PDA. The combination of PAK inhibitors with gemcitabine may be a more effective therapeutic approach in PDA treatment.
\end{abstract}

Correspondence to: Dr Mehrdad Nikfarjam or Dr Hong He, Department of Surgery, University of Melbourne, Austin Health, Studley Rd., Heidelberg, Victoria 3084, Australia

E-mail: m.nikfarjam@unimelb.edu.au

E-mail: hong.he@unimelb.edu.au

Abbreviations: PAK, p21 activated kinase; PDA, pancreatic ductal adenocarcinoma; Treg, T regulatory cells; MDSC, myeloid-derived suppressor cells; TILs, tumour-infiltrating leukocytes

Key words: p21 activated kinase, pancreatic cancer, tumour immune response, gemcitabine

\section{Introduction}

Pancreatic cancer is an aggressive and lethal malignancy with an overall 5-year survival rate $<8 \%$, and its death rate continues to increase by $0.3 \%$ per annum (1). Although surgery remains a potentially curative treatment, chemotherapy is an important and indispensable therapy in maximizing the life span for patients with both resectable and unresectable tumours. Currently, gemcitabine-based therapies, either alone or in combination with agents as such nab-paclitaxel (2), are favored approaches for treatment of patients with locally advanced or metastatic pancreatic cancer. Although the regimen, folic acid, 5-fluorouracil, irinotecan, oxalaplatin (FOLFIRINOX), has increased survival compared to gemcitabine, it often cannot be tolerated due to its high toxicity and the poor performance status of patients (3). This necessitates a continued search for more effective and less toxic combination therapies for the treatment of pancreatic cancer.

Immunotherapy to activate antitumour immunity has delivered promising results in various tumours (4). However, immunotherapy has little effect in pancreatic ductal adenocarcinoma (PDA) because of the potently immunosuppressive microenvironment in which immune cells, PDA cells and stroma interact to facilitate cancer progression (5). A combination approach involving chemotherapy, immunotherapy, targeted therapy against stromal elements, and other modalities, may be required in order to stimulate antitumour immunity, increase treatment efficacy and improve survival.

The p21 activated kinase (PAK) family of serine/threonine kinases, the downstream effector proteins of Rho GTPases, are categorized into two groups. Both group I (PAK1-3) and group II (PAK4-6) PAKs are involved in multiple cellular signaling pathways that regulate cell survival, proliferation, and migration (6). Overexpression and hyper-activation of PAKs contribute significantly to the initiation and progression of human cancers, and PAK1 and PAK4 are the best-studied members in this area. PAK1 expression is upregulated in both human pancreatic cancer cells and tissues (7). Inhibition of PAK1 in pancreatic cancer cell lines by shRNA knock-down or a PAK1 specific inhibitor (Frax-597) decreased cancer cell growth, synergistically with gemcitabine (7). PAK4 upregulation enhanced pancreatic cancer cell proliferation and survival through AKT- and ERK-dependent activation of the NF- $\kappa \mathrm{B}$ pathway (8), and stimulated migration and invasiveness. In addition, PAK4 has been linked to the maintenance of a 
stem cell-like phenotype (9) and is a predictive marker of gemcitabine sensitivity in pancreatic cancer cells (10).

Kras mutations have been observed in $>95 \%$ of PDA (11). The failure to identify a drug-binding domain in the Kras protein has made it difficult to be targeted therapeutically. Therefore, the ability to target other downstream molecules in Kras signaling pathways has become increasingly important. PAK1 can be activated via a Ras-dependent pathway and at least in part, mediates the effect of activated Kras in pancreatic cancer (6). In this study, a PAK inhibitor [PF-3758309, $\mathrm{IC}_{50}, 13.7 \mathrm{nM}$ for PAK1 and $1.3 \mathrm{nM}$ for PAK4 (12)] was used to evaluate the combined effect of PAK inhibition with gemcitabine on pancreatic cancer growth and on the tumour immune response.

\section{Materials and methods}

Cells and reagents. Human PANC-1 and MiaPaCa-2 pancreatic cancer cells (purchased from Sigma-Aldrich, Melbourne, Australia) and a murine PAN02 pancreatic cancer cell [obtained from National Cancer Institute (Frederick, MD, USA)] were cultured in Dulbecco's modified Eagle's medium (DMEM) supplemented with 5\% FBS (fetal bovine serum; Hyclone Laboratories Inc., Melbourne, Australia) in a $37^{\circ} \mathrm{C}$ incubator with a humidified atmosphere of $5 \% \mathrm{CO}_{2}$. PF-3758309 was purchased from Active Biochemical Co. Maplewood, NJ, USA and gemcitabine from Sigma-Aldrich. PAK1 knock-down (KD) PANC-1 and MiaPaCa-2 cells were generated by transfection with SureSilencing shRNA plasmids for human PAK1 (SABioscience, Doncaster, Australia), or with a scrambled sequence as a negative control, using Lipofectamine 2000 (Invitrogen, Mulgrave, Australia), according to the manufacturer's instructions. Stable clones were selected with geneticin (G418; $1 \mathrm{mg} / \mathrm{ml})$. PAK protein expression was detected by western blotting.

Cell proliferation. Cell proliferation was measured using a ${ }^{3} \mathrm{H}$-thymidine incorporation assay. Cells $\left(5 \times 10^{3}\right.$ cells/well $)$ were seeded in a 96-well plate and incubated in DMEM containing $5 \% \mathrm{FBS}$, with $1 \mu \mathrm{Ci} /$ well [methyl- ${ }^{3} \mathrm{H}$ ]-thymidine (Perkin-Elmer, Boston, MA, USA) in the presence of PF-3758309 at the concentrations indicated in the Results section. After $24 \mathrm{~h}$ cells were harvested using a NUNC cell harvester (Nunc, Roskilde, Denmark). The amount of ${ }^{3} \mathrm{H}$-thymidine incorporated through DNA synthesis was detected with a $\beta$-counter (Packard, Meriden, CT, USA). For combinational treatment, cells were pre-treated with PF-3758309 for $24 \mathrm{~h}$, and then PF-3758309 was removed. The cells were incubated with increasing concentrations of gemcitabine and $1 \mu \mathrm{Ci} /$ well [methyl- ${ }^{3} \mathrm{H}$ ]-thymidine for a further $24 \mathrm{~h}$ and harvested and detected as above.

Migration/invasion assay. Cell migration/invasion was measured using a Transwell Boyden Chamber assay as described previously (13). Membranes (8- $\mu \mathrm{m}$ pore size; Becton-Dickinson, NJ, USA) were coated with $3 \mu \mathrm{g}$ of human fibronectin on the lower surfaces and placed into a 24-well plate containing $600 \mu \mathrm{l} /$ well of serum-free DMEM with $0.1 \%$ bovine serum albumin (BSA). Cells were added to the upper chambers at $5 \times 10^{4} / 100 \mu 1$ with or without PF-3758309 at the concentrations indicated. Cells were then incubated for $24 \mathrm{~h}$. Cells that had not penetrated were removed from the upper surface by wiping with a cotton swab. The membranes were then fixed and stained with Quick-Dip (Fronine, Sydney, Australia). The cells that had migrated to the lower surface of the membranes were counted in 24-48 fields, depending on the cell line, at x40 magnification using a Nikon Coolscope (Coherent Scientific, Adelaide, Australia). The combined effects of gemcitabine and PF-3758309 were evaluated using the Chou-Talalay method as previously described (14). The combination index (CI) was calculated with the CalcuSyn program (T.C. Chou and M.P. Hayball; Biosoft, Cambridge, UK) using the mutually non-exclusive $(\alpha=1)$ isobologram equation. The CI value is interpreted as follows: $<1.0$, synergistic; 1.0, additive and $>1.0$, antagonistic.

Anchorage-independent assay. Anchorage-independent growth of pancreatic cancer cell lines was determined by soft agar assay as described previously (15). Briefly, cells $(2,000 /$ well $)$ were seeded in top agar layer containing $0.4 \%$ bacto-agar/DMEM $(0.5 \mathrm{ml})$ over a bottom agar layer of $0.6 \%$ bacto-agar/DMEM $(1 \mathrm{ml})$ in 12 -well plates. Both layers of soft agar were allowed to set at room temperature for 20-30 min before $0.5 \mathrm{ml}$ of DMEM containing treatment reagents was added to the wells. For wild-type MiaPaCa-2 and PANC-1 cells were divided into four groups: control, PF-3758309, gemcitabine, and combination of PF-3758309 with gemcitabine. For PAK1 knock-down MiaPaCa-2 and PANC-1, each cell line was divided into two groups: control and gemcitabine. The concentrations of PF3758309 and gemcitabine were chosen close to the $\mathrm{IC}_{50}$ values from the proliferation assay. Cell colonies were grown in a humidified $5 \% \mathrm{CO}_{2}$ incubator at $37^{\circ} \mathrm{C}$ for 4 weeks. Culture medium containing treatment reagents was changed twice weekly. At the endpoint, colonies were fixed and stained with $4 \%$ formalin and $0.005 \%$ crystal violet in phosphate-buffered saline (PBS). Colonies were counted manually and images were captured using a Leica microscope at $\mathrm{x} 4$ magnification.

Animal experiments. All mouse experiments were approved by the Austin Health Research Ethics Committee (A2013/04898). An orthotopic pancreatic cancer tail model was used to assess tumour growth in the pancreatic tails of C57BL/6 mice as previously described (16). PAN02 (a murine pancreatic cancer cell line) cells were injected into the pancreatic tails of 24 PAK1 wild-type (WT) mice, which were allocated randomly into four treatment groups (6 mice /group): control, intraperitoneal (i.p.) injection of saline every other day; gemcitabine $(40 \mathrm{mg} / \mathrm{kg})$, i.p. twice weekly; PF-3758309 (25 mg/kg), i.p. every other day; combination of gemcitabine with PF-3758309, following the individual treatment as described above. For assessment of tumour growth in PAK1 knockout (KO) C57BL/6 mice, 9 mice were injected with PAN02 cells in the pancreatic tail and were allocated randomly into two groups: control $(n=4)$ and gemcitabine $(n=5)$, and treated as described above. All treatments commenced one week after surgical induction and continued for 4 weeks. All mice were monitored based on strict health monitoring criteria and euthanized when a poor health score was reached. At the endpoint, tumour dimensions were measured using micro-calipers. The tumour volume was 

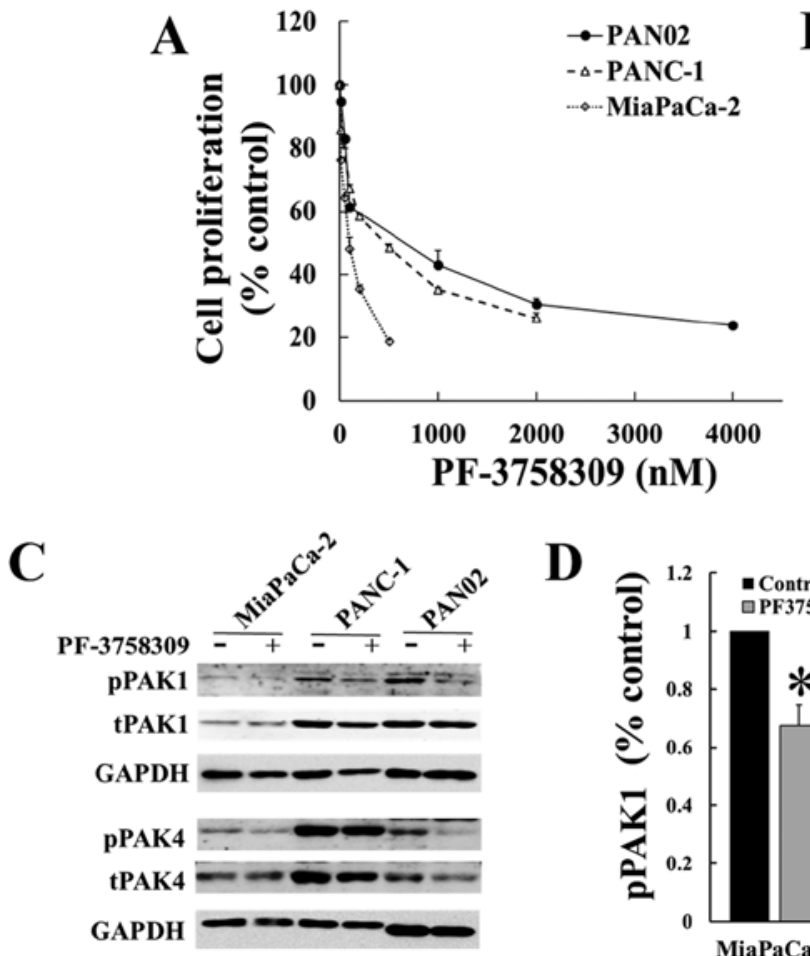
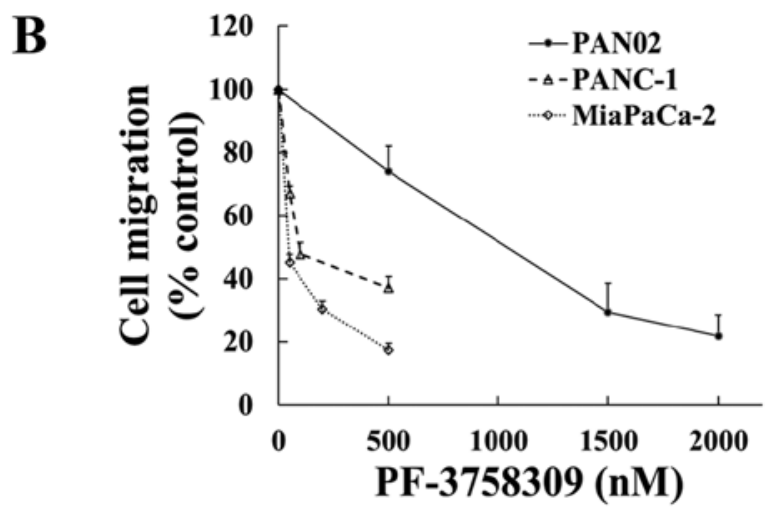

$\mathbf{E}$

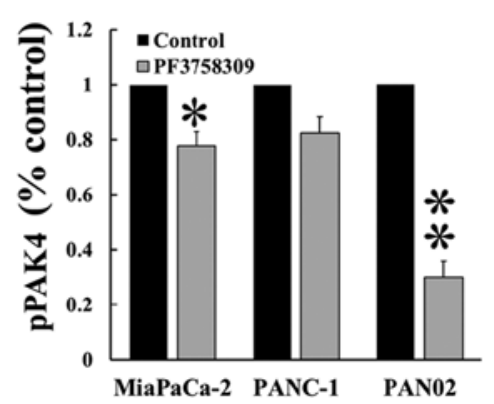

Figure 1. PF-3758309 inhibits proliferation and migration/invasion of pancreatic cancer cells by reducing the activity of PAK1 and/or PAK4. Pancreatic cancer cells were treated with PF-3758309 at the indicated doses for $24 \mathrm{~h}$. Cell proliferation and migration/invasion were measured by ${ }^{3} \mathrm{H}$-thymidine incorporation and Boyden chamber Transwell assay, respectively. PF-3758309 inhibited the proliferation (A) and migration/invasion (B) of MiaPaCa-2, PANC-1 and PAN02 pancreatic cancer cells in a dose-dependent manner. When cells were incubated with PF-3758309 at the $\mathrm{IC}_{50}$ concentration calculated from (A) (values given in Table I) for $24 \mathrm{~h}$, the levels of active phosphorylated PAK1 (pPAK1) and PAK4 (pPAK4) were significantly decreased. The ${ }^{3} \mathrm{H}$-thymidine values (A) and the penetrated cell numbers (B) from control were taken as 100\%. The data were summarized from three independent experiments. (C-E) PF-3758309 inhibited the activities of both PAK1 and PAK4 in pancreatic cancer cell lines. ${ }^{*} \mathrm{p}<0.05,{ }^{* *} \mathrm{p}<0.01$, compared to the values from cells without any treatment.

calculated by using the formula: $\mathrm{V}=\mathrm{L} \times \mathrm{W} \times \mathrm{H} \times(\pi / 6)$, where $\mathrm{L}$ was the length; $\mathrm{W}$ the width; and $\mathrm{H}$ the height.

Flow cytometric analysis. Spleens were harvested from tumour-bearing mice as described above, and homogenised in $0.5 \%$ BSA in PBS. The splenic cells were collected by centrifugation after red cells had been removed using red cell lysis buffer (150 mM NH $4 \mathrm{Cl}, 10 \mathrm{mM} \mathrm{NaHCO} 3,0.1 \mathrm{mM}$ EDTA). After further washing in $0.5 \% \mathrm{BSA}$ in $\mathrm{PBS}$, the remaining cells were incubated for $1 \mathrm{~h}$ on ice with FITC- or APC-labelled antibodies against B220, CD3, CD8 (1:100) (BD Biosciences, North Ryde, Australia) or CD4 (1:10) (Miltenyi Biotec, Macquarie Park, Australia), or with respective isotype controls. Cells were washed 3 times in $0.5 \%$ BSA in PBS before analysis by FACSCanto II (BD Biosciences). Data were analysed using Weasel software (Cytometry Laboratory, Walter and Eliza Hall Institute, Parkville, Australia).

Immunohistochemistry stain. Tumour tissues, collected from the orthotopic tail model as described above, were embedded in paraffin and stained with Ki67 antibody (Thermo Fisher Scientific, Melbourne, Australia) for proliferation, active caspase 3 antibody (Cell Signaling Technology, Arundel, Australia) for apoptosis, or CD3 antibody (Abcam, Melbourne, Australia) for total $\mathrm{T}$ cell surface marker. Samples were imaged using a Leica microscope for at least 4 fields at $\mathrm{x} 40$ magnification. For the $\mathrm{CD}^{+}$stain, cell numbers were counted per $x 40$ field. For the Ki67 and caspase 3 stains, the ratio of positive cells to total number of cells in each field was calculated.

Western blotting. Cells were treated with PF-3758309 at the $\mathrm{IC}_{50}$ concentration for $24 \mathrm{~h}$, lysed in SDS sample buffer, and denatured by heating at $95^{\circ} \mathrm{C}$ for $5 \mathrm{~min}$. Proteins in the cell lysates were detected with antibodies against phospho-PAK1, PAK1, phospho-PAK4, PAK4 and GAPDH. All primary antibodies were purchased from Cell Signaling Technology. The bound antibodies were visualized using ECL reagents (GE Healthcare, Amersham, UK) and the density of each band was analyzed using Multigauge computer software (Berthold, Bundoora, Australia).

Statistical analysis. All values were expressed as mean \pm standard error. Results were analyzed by Student's t-test or one-way ANOVA (SPSS, IBM, New York, NY, USA). Differences between two means with $\mathrm{p}<0.05$ were considered statistically significant.

\section{Results}

PF-3758309 inhibits pancreatic cancer cell proliferation and migration. Human (MiaPaCa-2 and PANC-1) and murine (PAN02) pancreatic cancer cells were cultured with increasing doses of PF-3758309 (Fig. 1A and B) for $24 \mathrm{~h}$. Cell proliferation and migration/invasion were measured by ${ }^{3} \mathrm{H}$-thymidine incorporation and Boyden chamber assays, 
A

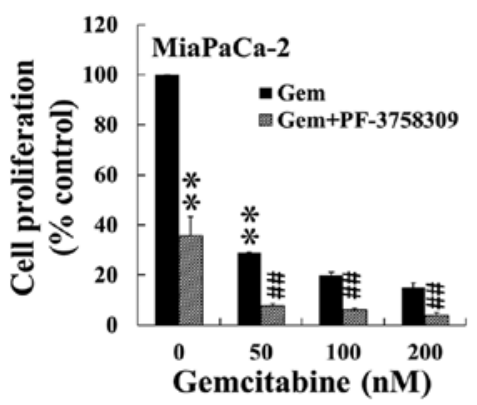

B

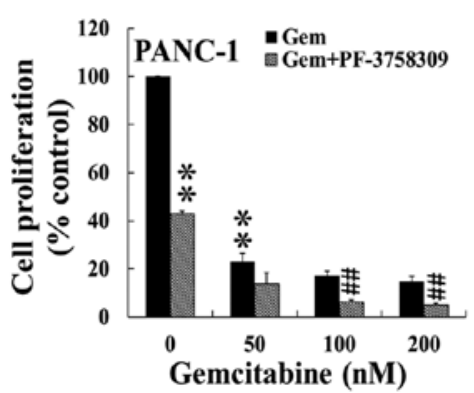

C

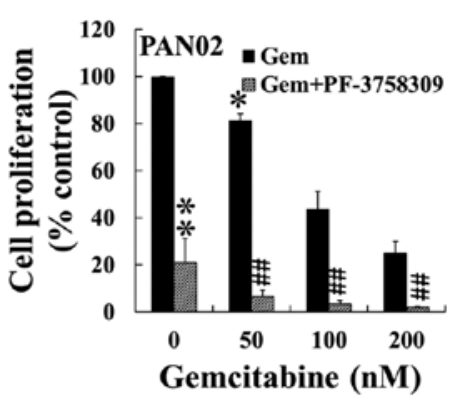

Figure 2. PF-3758309 and gemcitabine synergistically inhibits the proliferation of pancreatic cancer cells. The pancreatic cancer cell lines, MiaPaCa-2 (A), PANC-1 (B) and PAN02 (C) were pre-treated with PF-3758309 at the $\mathrm{IC}_{50}$ concentrations calculated from proliferation (Fig. 1 and Table I) for 24 h. PF-3758309 was then removed, and the cells were treated with gemcitabine (Gem) at 50,100 and $200 \mathrm{nM}$ for another $24 \mathrm{~h}$. Cell proliferation was measured by ${ }^{3} \mathrm{H}$-thymidine incorporation. PF-3758309 inhibited the proliferation of all three cell lines tested synergistically with gemcitabine. The data were summarized from three independent experiments. ${ }^{*} \mathrm{p}<0.05,{ }^{* *} \mathrm{p}<0.01$, compared to the values from cells without any treatment. ${ }^{\# \#} \mathrm{p}<0.01$, compared to the values from cells treated by either PF-3758309 or gemcitabine alone.

A

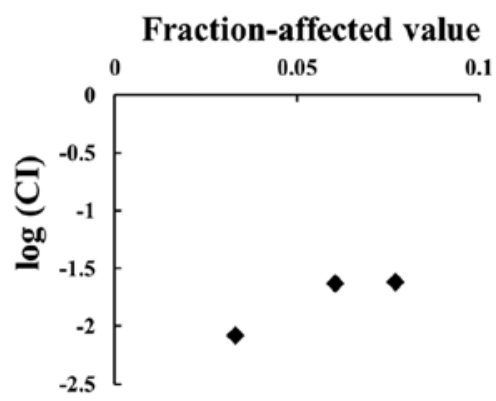

MiaPaCa-2
B

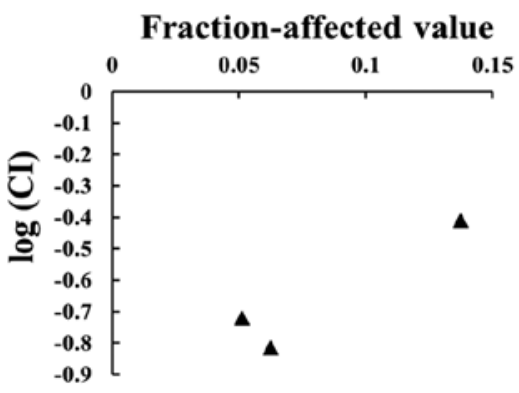

PANC-1
C

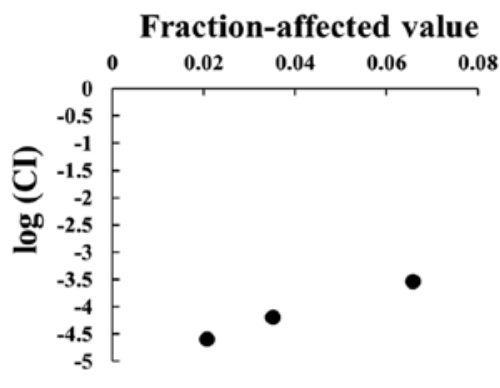

PAN02

Figure 3. PF-3758309 inhibits pancreatic cancer cell proliferation synergistically with gemcitabine. The pancreatic cancer cell lines MiaPaCa-2, PANC-1 (human) and PAN02 (murine) were pre-treated with PF-3758309 at the $\mathrm{IC}_{50}$ concentrations given in Table $\mathrm{I}$ for $24 \mathrm{~h}$ followed by incubation with the concentrations of gemcitabine indicated in Fig. 2 for another $24 \mathrm{~h}$. Cell proliferation was measured by ${ }^{3} \mathrm{H}$-thymidine incorporation. The combined effects of gemcitabine and PF-3758309 were evaluated by calculating the combination index (CI) using the mutually non-exclusive $(\alpha=1)$ isobologram equation. The CI value is interpreted as follows: $<1.0$, synergistic; 1.0 , additive and $>1.0$, antagonistic.

respectively. PF-3758309 decreased the proliferation and migration/invasion of both human and murine pancreatic cancer cells (Fig. 1A and B) in a dose-dependent manner, the $\mathrm{IC}_{50}$ values are given in Table $\mathrm{I}$. The three pancreatic cancer cell lines were then incubated for $24 \mathrm{~h}$ with PF-3758309 at the $\mathrm{IC}_{50}$ value calculated from the proliferation assays (Fig. 1A), and the expression of phosphorylated and active PAK1 and PAK4, as well as total PAK1 and PAK4, was measured by western blotting. PF-3758309 inhibited the activity of PAK1 in the three cell lines tested (Fig. 1C), and the activity of PAK4 in MiaPaCa-2 and PAN02. These data indicated that PF-3758309 supressed pancreatic cancer cell proliferation and migration probably via inhibition of the activities of both PAK1 and PAK4.

PF-3758309 inhibits pancreatic cancer cell proliferation synergistically with gemcitabine. The combined effects of PF-3758309 and gemcitabine on proliferation were measured by incubation of the pancreatic cancer cell lines with different concentrations of gemcitabine, alone or in combination with pre-treatment of PF-3758309, at concentrations around the $\mathrm{IC}_{50}$ values given in Table I). Gemcitabine on its own inhibited proliferation in the three pancreatic cancer cell lines in a
Table I. The $\mathrm{IC}_{50}$ for inhibition of proliferation and migration/ invasion of pancreatic cancer cell lines by PF-3758309.

\begin{tabular}{lccc}
\hline & MiaPaCa-2 & PANC-1 & PAN02 \\
\hline Proliferation (nM) & $87+15 \mathrm{nM}$ & $500+70 \mathrm{nM}$ & $805+155 \mathrm{nM}$ \\
Migration/invasion (nM) & $60+10 \mathrm{nM}$ & $141+35 \mathrm{nM}$ & $1230+220 \mathrm{nM}$ \\
\hline
\end{tabular}

dose-dependent manner (Fig. 2). A further reduction of proliferation was observed in all three cell lines when the cells were treated with PF-3758309 and gemcitabine in combination, compared to treatment with gemcitabine alone (Fig. 2). The combined effect of PF-3758309 and gemcitabine was shown to be synergistic rather than additive when analysed by the Chou-Talalay method (17) (Fig. 3). These results demonstrated that PF-3758309 and gemcitabine synergistically inhibited pancreatic cancer cell growth.

Inhibition of PAKs promotes the suppression by gemcitabine of anchorage-independent growth of pancreatic cancer cells. 
A

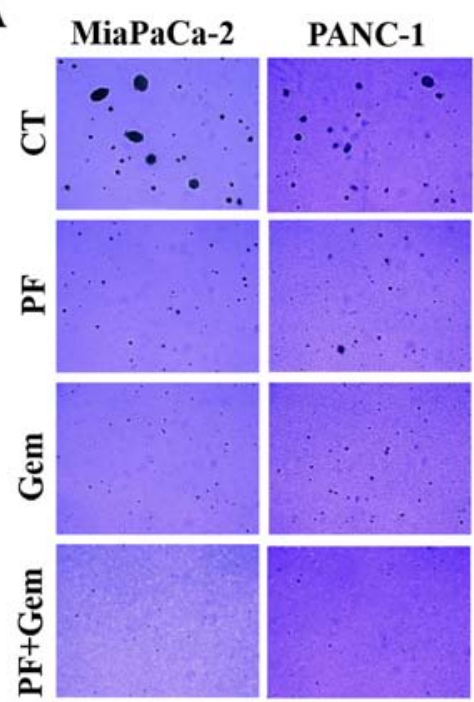

B

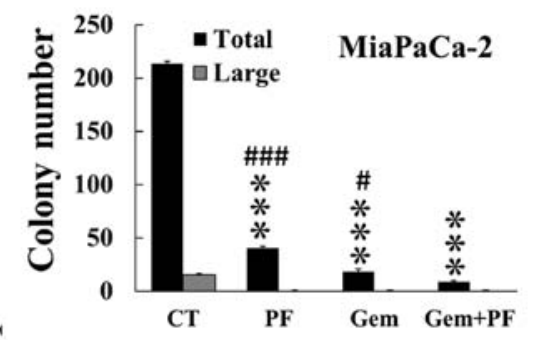

C

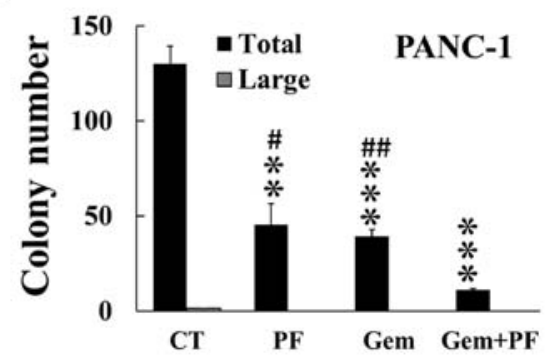

Figure 4. PF-3758309 and gemcitabine synergistically inhibits the anchorage-independent growth of pancreatic cancer cells. The MiaPaCa-2 and PANC-1 cell lines (2,000 cells/dish) were seeded in agar with or without PF-3758309 [PF, $80 \mathrm{nM}$ (MiaPaCa-2) or $500 \mathrm{nM}$ (PANC-1)] plus or minus gemcitabine (Gem, $50 \mathrm{nM}$ ), and incubated for 4 weeks with the top media changed twice a week. One representative data set from three independent experiments is presented. Colonies containing numbers of cells $>100$ were considered as large. ${ }^{* *} \mathrm{p}<0.01,{ }^{* * *} \mathrm{p}<0.001$, compared to the control (CT) values. ${ }^{\#} \mathrm{p}<0.05,{ }^{\# \#} \mathrm{p}<0.01$, compared to the values from cells treated with both PF-3758309 and gemcitabine.

A
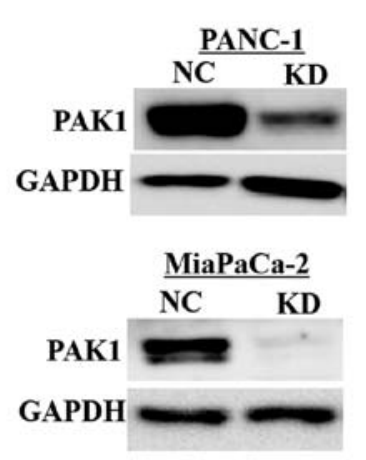

B

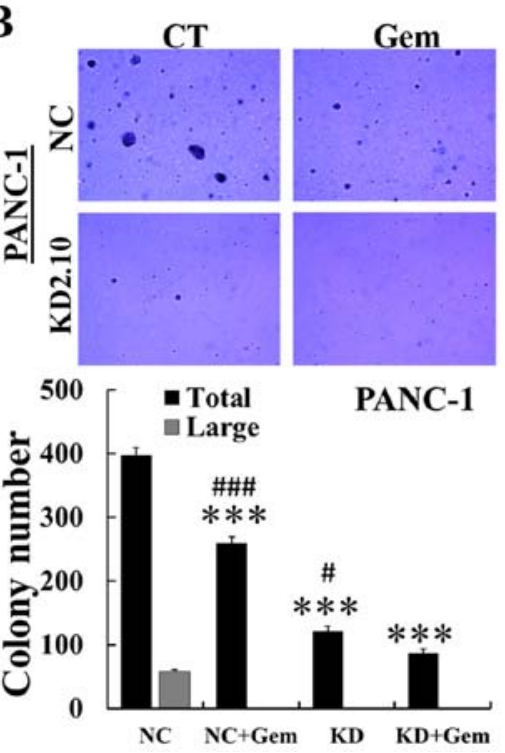

C
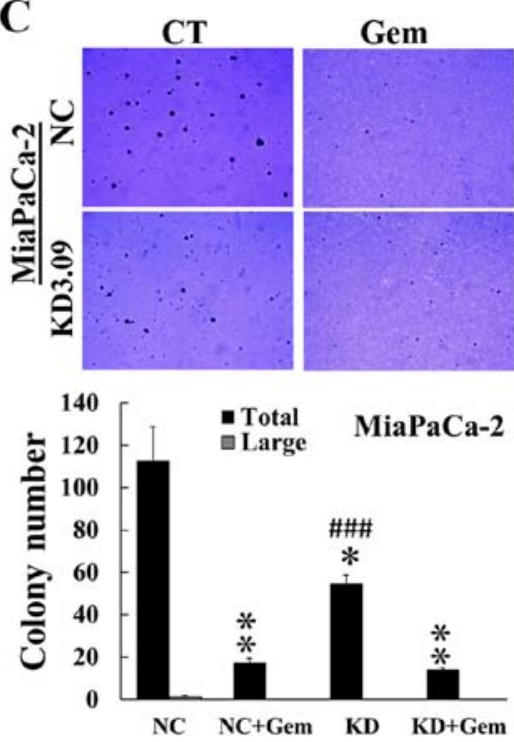

Figure 5. PAK1 knock-down supresses the anchorage-independent growth of pancreatic cancer cells and promotes the inhibitory effect of gemcitabine. PAK1 knock-down (KD) or negative control (NC) clones of PANC-1 or MiaPaCa-2 cells (2,000/dish) were seeded in agar with or without gemcitabine (Gem, $50 \mathrm{nM}$ ), and incubated for 4 weeks with the top media changed twice a week. One representative data set from three independent experiments is presented. Colonies containing $>100$ cells were considered as large. ${ }^{*} \mathrm{p}<0.05,{ }^{* *} \mathrm{p}<0.01,{ }^{* * *} \mathrm{p}<0.001$, compared to the values from the NC control (CT). ${ }^{\#} \mathrm{p}<0.05,{ }^{\# \#} \mathrm{p}<0.01$, compared to the values from PAK1 KD cells treated with gemcitabine.

The combined effect of inhibition of PAK by PF-3758309 with gemcitabine on anchorage-independent growth of pancreatic cancer cells was measured by growing cells in soft agar, followed by treatment with gemcitabine alone or in combination with PF-3758309 at concentrations around the $\mathrm{IC}_{50}$ values calculated from the proliferation assay. Either PF-3758309 or gemcitabine alone decreased the numbers of colonies formed in the agar in both MiaPaCa-2 and PANC-1 cells (Fig. 4). Combined treatment with PF-3758309 and gemcitabine resulted in a significant further decrease in the numbers of colonies formed compared with either PF-3758309 or gemcitabine alone (Fig. 4). PAN02 did not grow in the soft agar under current condition.

In order to confirm this result, PAK1 expression was reduced by knockdown (KD) with shRNA (Fig. 5A), and the numbers of colonies formed in PAK1 KD with or without gemcitabine were analysed. Cells transfected with a scrambled shRNA sequence served as a negative control (NC). The number of colonies formed in PAK1 KD cells was significantly reduced compared to NC cells. Gemcitabine treatment 


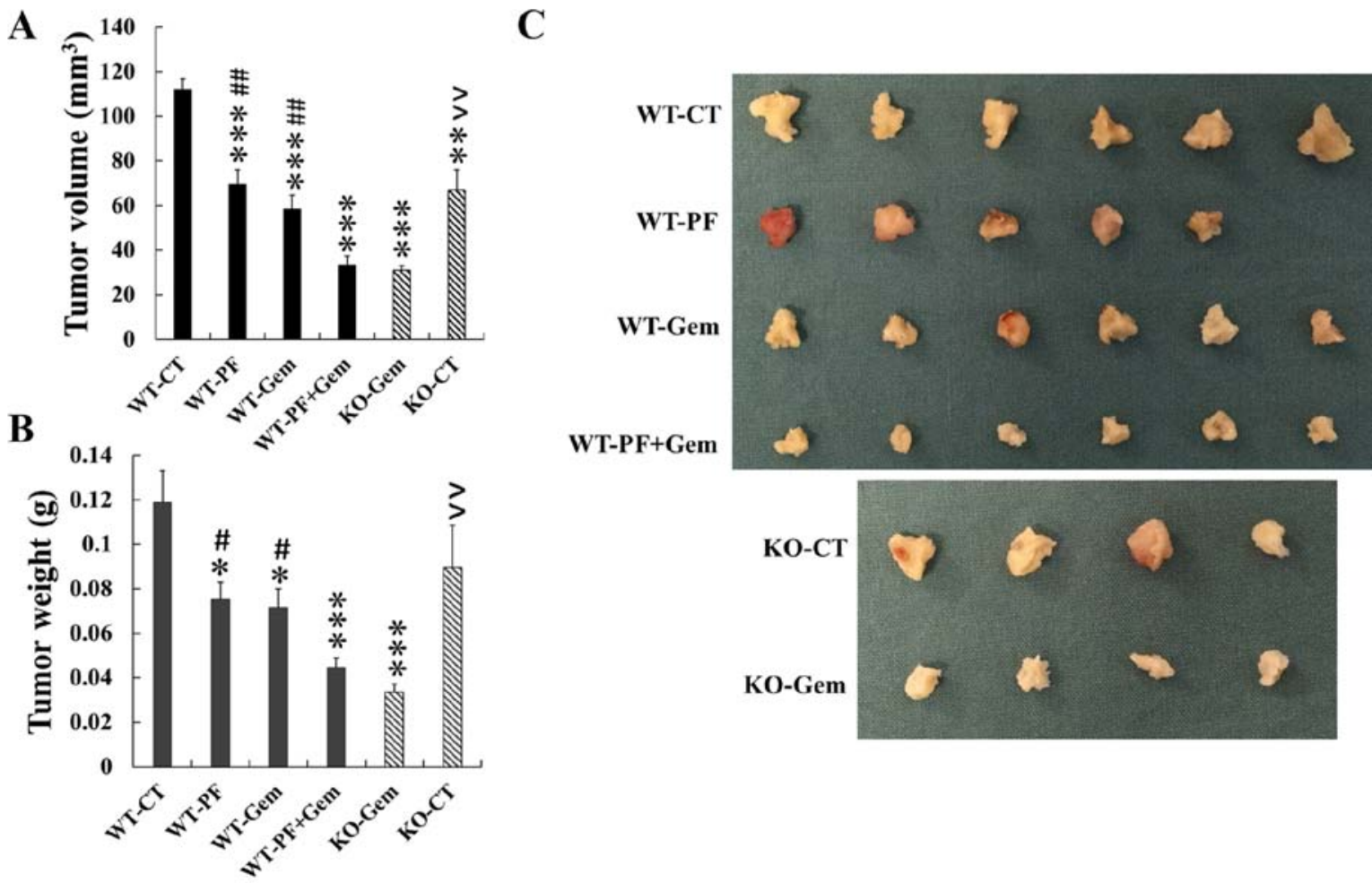

Figure 6. Inhibition of PAK enhances the antitumour effect of gemcitabine on pancreatic cancer growth in an orthotopic cancer model. Twenty-four

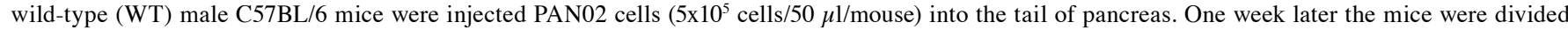
into 4 groups, 6 per group, and were treated by intra-peritoneal injection with 5\% DMSO saline (CT, control), PF-3758309 (PF, 25 mg/kg), gemcitabine $(\mathrm{Gem}, 40 \mathrm{mg} / \mathrm{kg}$ ), or PF plus Gem for 4 weeks. Nine PAK1 knockout (KO) mice were subjected to similar procedure. The PAK1 KO mice were divided into control $(\mathrm{CT}, \mathrm{n}=4)$ and gemcitabine-treated $(\mathrm{Gem}, \mathrm{n}=5)$ groups. The mice were sacrificed at the endpoint, their pancreata isolated, and tumour volume $(\mathrm{A})$ and weight (B) were measured. Tumour images were taken (C). ${ }^{*} \mathrm{p}<0.05,{ }^{* *} \mathrm{p}<0.01,{ }^{* * *} \mathrm{p}<0.001$, compared to the values from WT control mice. ${ }^{\#} \mathrm{p}<0.05,{ }^{\# \#} \mathrm{p}<0.01$, compared to the values from WT mice treated with PF plus Gem, ${ }^{\wedge} \mathrm{p}<0.01,{ }^{\wedge \wedge} \mathrm{p}<0.001$, compared to the values from PAK1 KO mice treated with Gem.

further decreased the colony numbers in PANC-1 PAK1 KD cells (Fig. 5B), but not in MiaPaCa-2 PAK1 KD cells (Fig. 5C). Furthermore, large colonies containing $>100$ cells only formed in untreated $\mathrm{NC}$ cells. These data demonstrated that inhibition of PAKs by PF-3758309 or by shRNA knockdown enhanced the suppressive effect of gemcitabine on anchorage-independent growth of pancreatic cancer cells.

Inhibition of PAKs sensitizes the response of pancreatic cancers to gemcitabine in vivo. A mouse orthotopic pancreatic cancer model was used to determine the effect of inhibition of PAKs on the response of pancreatic cancers to gemcitabine treatment in vivo. Tumours were induced by injection of pancreatic cancer cells into the tail of the pancreas as previously described (16). The tumour take rate was $100 \%$ in both PAK1 wild-type (WT) and PAK1 knockout (KO) C57BL/6 mice. None of the mice died from surgery. In PAK1 WT mice, either PF-3758309 or gemcitabine alone significantly reduced tumour growth by decreasing the tumour volume (Fig. 6A and C) and tumour weight (Fig. 6B), compared to untreated controls. A further reduction in both tumour volume and weight was observed in mice treated with the combination of PF-3758309 and gemcitabine. The tumour volume, but not the tumour weight, was significantly decreased in PAK1 KO mice when compared to PAK1 WT mice. Gemcitabine treatment of PAK1 KO mice caused a further reduction in both tumour volume and tumour weight when compared to the tumours in untreated PAK1 KO mice.
Either PF-3758309 or gemcitabine alone inhibited tumour growth by decreasing cell proliferation as measured by Ki67 immunohistochemistry (Fig. 7A). The proliferation in tumours from PAK1 WT mice treated with either PF-3758309 or gemcitabine was reduced to $60.6 \%$ and $60.1 \%$ of that in tumours from untreated mice. The combination of PF-3758309 and gemcitabine further reduced the proliferation to $25.9 \%$ of the untreated PAK1 WT mice. Importantly, the proliferation in tumours from PAK1 KO mice was also significantly reduced compared to tumours from untreated PAK1 WT mice. Gemcitabine treatment of PAK1 KO mice further decreased cell proliferation in tumours when compared to the corresponding untreated PAK1 KO mice (Fig. 7A). No significant difference was detected in apoptosis in tumours from all six groups although compared to control of PAK1 WT mice, there were increased trend in all other groups of treatment mice (Fig. 7B). These results indicate that inhibition of PAKs by PF-3758309 or by PAK1 knockout sensitized pancreatic cancers to gemcitabine in vivo at least by decreasing cell proliferation.

Inhibition of PAKs enhances tumour immune response in vivo. To determine the effect of PAK inhibition on tumour immune response, spleens were dissected from the tumour-bearing mice treated as shown in Fig. 6. The red blood cells were removed, and the splenic cells were extracted and subjected to FACS analysis as described in the Materials and methods. In the PAK1 WT mice, both $\mathrm{CD} 3^{+}$and $\mathrm{CD} 8^{+} \mathrm{T}$ lymphocytes 
A
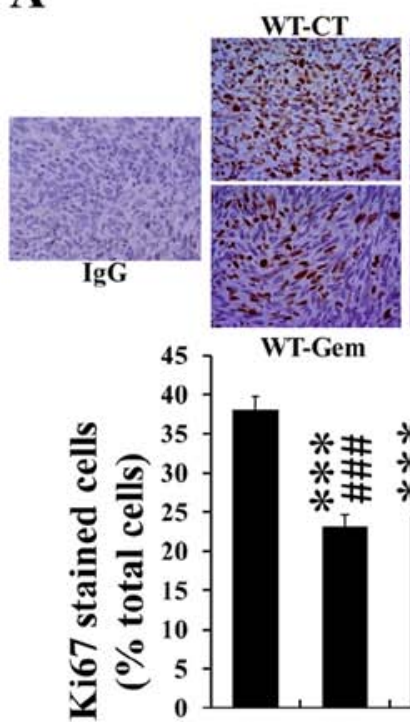

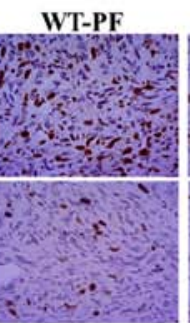

WT-PF+Gem

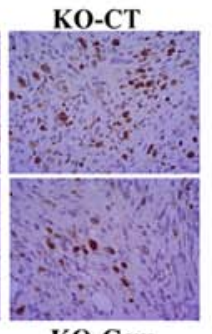

KO-Gem

B
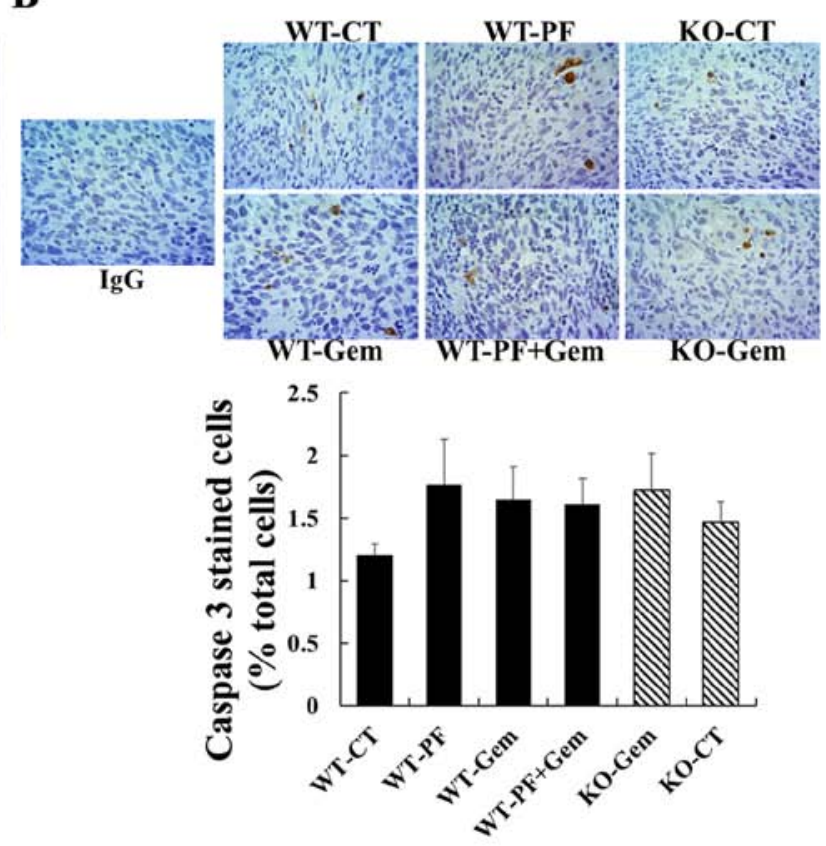

Figure 7. Inhibition of PAK promotes the inhibitory effect of gemcitabine on tumour proliferation. Tumour samples obtained from mice treated as in Fig. 6 , were fixed and tumour cell proliferation (A) and apoptosis (B) were determined by immunohistochemistry stain of Ki67 (A) and caspase 3 (B). ${ }^{* * *}$ p $<0.001$, compared to the values from PAK1 wild-type (WT) control mice. ${ }^{\# \# \# ~} \mathrm{p}<0.001$, compared to the values from WT mice treated with PF-3758309 (PF) plus gemcitabine $(\mathrm{Gem}),{ }^{\wedge \wedge} \mathrm{p}<0.001$, compared to the values from PAK1 knockout $(\mathrm{KO})$ mice treated with Gem.

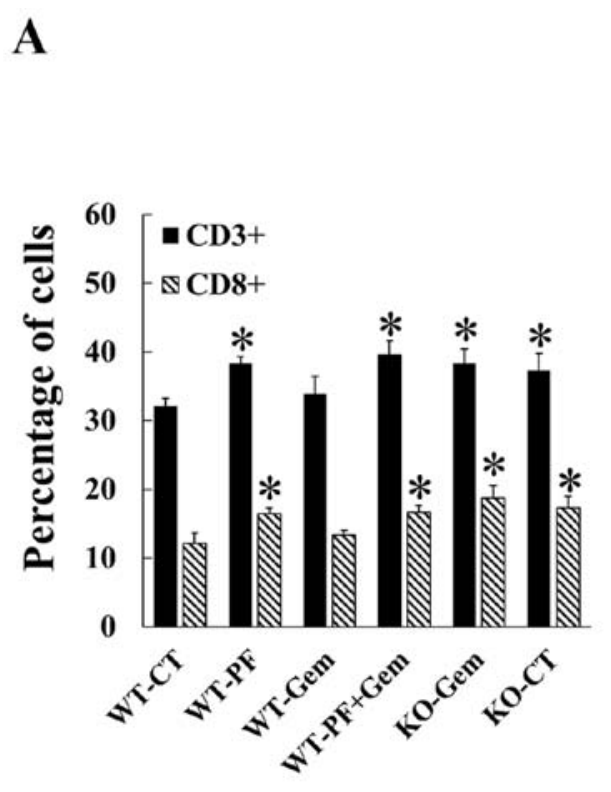

B
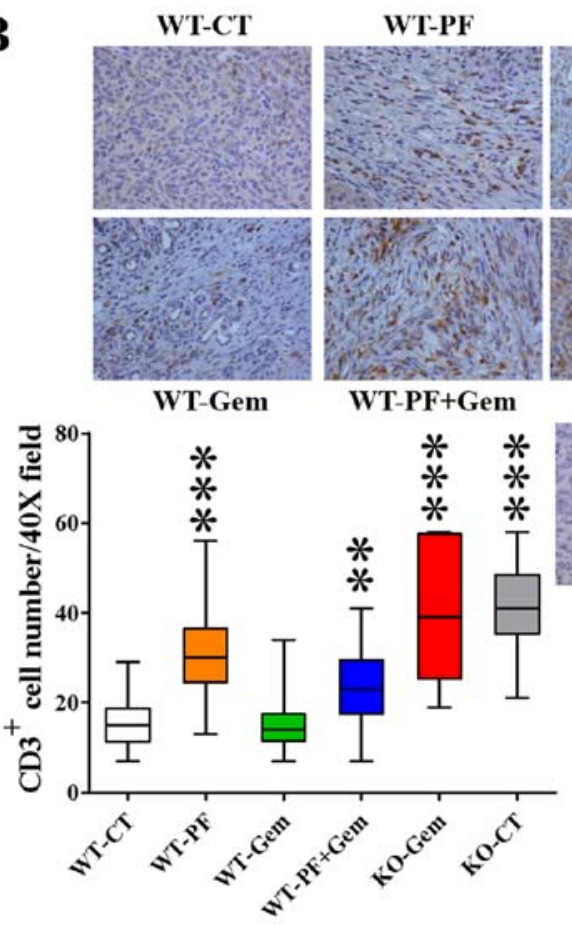

WT-PF
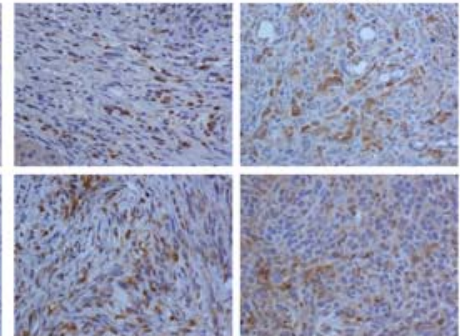

KO-Gem

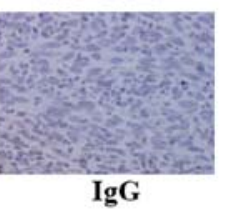

Figure 8. Inhibition of PAK upregulates the immune system of mice bearing pancreatic tumours and stimulates tumour immune infiltration. Fresh spleens were dissected out from the mice described in Fig. 5. The splenic lymphocytes were analysed by flow cytometry (A). The percentage of CD $3^{+}$or $\mathrm{CD} 8^{+}$cells was calculated using the Weasel computer program (Cytometry Laboratory, Walter and Eliza Hall Institute, Parkville, Australia). The pancreatic tumour tissues were taken from all 6 groups of mice and stained for $\mathrm{CD}^{+}$cells, which were then counted under the microscope at $\mathrm{x} 40$ magnification (B). WT, wild-type PAK1; KO, knockout PAK1; CT, control; Gem, gemcitabine; PF, PF-3758309. " $\mathrm{p}<0.05,{ }^{* *} \mathrm{p}<0.01,{ }^{* * * *} \mathrm{p}<0.001$, compared to the values from PAK1 WT control mice.

were increased by treatment with PF-3758309 alone or in combination with gemcitabine (Fig. 8A). Both $\mathrm{CD}^{+}$and $\mathrm{CD}^{+}$ $\mathrm{T}$ lymphocytes were increased in untreated PAK1 KO mice compared to untreated PAK1 WT mice. Gemcitabine treatment did not cause any significant changes in $\mathrm{CD}^{+}$or $\mathrm{CD}^{+}$ T lymphocytes of either PAK1 WT or PAK1 KO mice (Fig. 8A). 
$\mathrm{CD}^{+} \mathrm{T}$ lymphocytes were stained in pancreatic tumour tissues from all 6 groups of mice to determine the tumour-infiltrating immune cells. In the tumours in PAK1 WT mice, CD3 ${ }^{+}$ T lymphocytes were increased by treatment with PF-3758309 alone or in combination with gemcitabine (Fig. 8B). CD3 ${ }^{+}$ $\mathrm{T}$ lymphocytes were also increased in tumours in untreated PAK1 KO mice compared to tumours in untreated PAK1 WT mice. Gemcitabine treatment alone did not cause any significant changes in $\mathrm{CD}^{+} \mathrm{T}$ lymphocytes in tumours from either PAK1 WT or PAK1 KO mice (Fig. 8B). These results indicate that inhibition of PAKs by PF-3758309 or by PAK1 KO upregulated the immune response of tumour-bearing mice by stimulation of both splenic $\mathrm{CD}^{+}$and $\mathrm{CD}^{+} \mathrm{T}$ lymphocytes and tumour-infiltrating $\mathrm{CD}^{+} \mathrm{T}$ lymphocytes.

\section{Discussion}

Our most important findings in this study are that, in an orthotopic pancreatic cancer mouse model, inhibition of PAK1 and PAK4 by PF-3758309 or depletion of PAK1 not only suppressed the growth of pancreatic cancer in vivo, but also stimulated the immune response by increasing the numbers of splenic $\mathrm{CD}^{+}$and $\mathrm{CD}^{+} \mathrm{T}$ lymphocytes as well as by enhancing the tumour-infiltrating $\mathrm{CD}^{+} \mathrm{T}$ lymphocytes. Tumour-infiltrating leukocytes (TILs) are critical determinants of clinical outcome of cancer patients, and play key roles in cancer progression and in therapeutic responses (18-20). Among TILs, the activation and location of $\mathrm{T}$ lymphocytes are particularly important. The location and density of intratumoural $\mathrm{CD}^{+} \mathrm{T}$ lymphocytes alters their prognostic significance (21). Infiltration of $\mathrm{CD}^{+} \mathrm{T}$ lymphocytes is associated with a significantly higher rate of progression-free survival of gastrointestinal cancer patients (22). In PDA patients, the presence of both $\mathrm{CD}^{+}$and $\mathrm{CD}^{+} \mathrm{T}$ lymphocytes in tumour tissues correlated with an improved prognosis and increased 5-year survival rate (23-25). These pieces of evidence stimulated the initiation and development of immune-therapeutic approaches to enhance the antitumour immune response through regulation of TILs. Our finding that inhibition of PAK1 and PAK4 by PF-3758309 or depletion of PAK1 increased the number of the tumour infiltrating $\mathrm{CD}^{+} \mathrm{T}$ lymphocytes in an orthotopic pancreatic cancer mouse model indicates the potential role of PAK inhibitors in modulation of TILs in order to improve antitumour immunity. Our finding also prompted us to further investigate the role of PAKs in tumour immune response and tumour microenvironment using KPC mouse (Kras ${ }^{\mathrm{G} 12 \mathrm{D} /+}$; Trp53 ${ }^{\mathrm{R} 172 \mathrm{H} /+}$; Pdx-1-Cre) which incorporates expression of $\mathrm{Kras}^{\mathrm{G} 12 \mathrm{D} /+}$ and $\operatorname{Trp} 53^{\mathrm{R} 172 \mathrm{H} /+}$ alleles targeted to the mouse pancreas by Cre recombinase under the control of the pancreas-specific Pdx-1 promoter and reproduces key features of human PDA (26).

Gemcitabine, as the base-line therapeutic agent for pancreatic cancer, has been reported to reduce the number of immunosuppressive $\mathrm{T}$ regulatory cells (Treg) and myeloidderived suppressor cells (MDSC), while restoring the ratio of $\mathrm{T}$ effector cells to Treg in the blood of pancreatic cancer patients (27). When comparing the number of intratumoural immune cells from patients undertaking gemcitabine treatment to the non-treated controls, gemcitabine did not change the number of intratumoural $\mathrm{CD}^{+} \mathrm{T}$ lymphocytes, but increased the ratios of $\mathrm{CD}^{+} /$Treg and $\mathrm{CD}^{+} /$Treg, possibly by reducing the number of $\mathrm{FOXP}^{+}$cells (28). In agreement with these clinical observations in patients, in our orthotopic pancreatic cancer model in mice, gemcitabine on its own did not change the number of either splenic $\mathrm{CD}^{+}$or $\mathrm{CD}^{+} \mathrm{T}$ lymphocytes or of intratumoural $\mathrm{CD}^{+} \mathrm{T}$ lymphocytes (Fig. 8). The effect of gemcitabine on Treg and MDSC in our model remains to be determined.

Although targeted therapies and immunotherapy have significantly improved the outcome of many solid malignancies such as melanoma and lung cancer, no such effective therapy has been developed for PDA, despite the knowledge of key mutations and an increasing understanding of the tumour microenvironment. Current treatment options for PDA mainly involve combination cytotoxic chemotherapies, which provide a marginal survival benefit at the cost of significant toxicity. Gemcitabine-based treatment is still most commonly applied to pancreatic cancer patients because of its general good patient tolerability and lack of better alternative less toxic combinations of chemotherapies (29). Indeed the combination of targeted therapies of gemcitabine, combined with agents, such as cetuximab or bevacizumab, has not added any additional benefit $(30,31)$.

Consistent with previous reports, we have shown that inhibition of both PAK1 and PAK4 by PF-3758309 suppressed the growth and migration/invasion of pancreatic cancer cells. Although the Boyden chamber assay is recognised as a standard method to measure cell migration/invasion (in the case of cancer cells). The measurement of MMP-2 and MMP-9 will certainly strengthen our data here, which will be added in our future study. Further we have demonstrated that PF-3758309, either on its own or synergistically with gemcitabine, reduced the growth of pancreatic cancer both in vitro and in vivo. The inhibitory effect of PF-3758309 on three cell lines tested seems to be correlated well with the activity of PAK1 as MiaPaCa-2 with lowest level of active phosphorylated PAK1 (pPAK1) responded to PF-378309 most sensitively while PAN02 with highest level of pPAK1 responded to PF-3758309 least sensitively (Fig. 1 and Table I). That the inhibitory effect of PF-3758309 is mainly mediated by a PAK1-dependent pathway is clearly demonstrated by our in vivo observation in the orthotopic pancreatic cancer model that tumour growth was inhibited by PF-3758309 in PAK1 wild-type mice to a similar degree to the tumour growth inhibition by PAK1 depletion in PAK1 knockout mice (Fig. 6). When determining the hematologic toxicities by full blood count, PF-3758309 and gemcitabine alone or in combination reduced the haemoglobin by $10 \%$ while PF-3758309 increased the platelet and neutrophils by $50 \%$ and $110 \%$, respectively (data not shown). PF-3758309 and/or gemcitabine did not affect any other hematologic index tested. Our findings that inhibition of PAKs not only suppressed pancreatic cancer growth (both alone and synergistically with gemcitabine), but also upregulated the tumour immune response by increasing the splenic and intratumoural lymphocytes, make PAKs in general, and PAK1 in particular, an attractive target(s) in developing effective therapeutic regimen.

In conclusion, our results that inhibition of PAK1 and PAK4 suppressed pancreatic cancer growth/invasion, stimulated the tumour immune response, and promoted the inhibitory effect of gemcitabine, indicate the potential role of PAKs in the 
immune response to pancreatic tumours, and provide a solid base for the development of a combination treatment of PAK targeted therapy with gemcitabine to improve the outcome for PDA patients.

\section{Acknowledgements}

The authors would like to thank Professor Mauro S. Sandrin for help in the flow cytometry analysis. This study was supported by grants from the National Health and Medical Research Council of Australia (1041831, GSB/AS), and the Pancare Foundation (GSB, MN). H.H. was supported by Baldwin trust fund. K.W. wass supported by Melbourne International Fee Remission Scholarship (MIFRS), Melbourne International Research Scholarship (MIRS) and Pancare Foundation (Moshe Sambor Scholarship).

\section{References}

1. Siegel RL, Miller KD and Jemal A: Cancer Statistics, 2017. CA Cancer J Clin 67: 7-30, 2017.

2. Von Hoff DD, Ervin T, Arena FP, Chiorean EG, Infante J, Moore M, Seay T, Tjulandin SA, Ma WW, Saleh MN, et al: Increased survival in pancreatic cancer with nab-paclitaxel plus gemcitabine. N Engl J Med 369: 1691-1703, 2013.

3. Zhang Y, Hochster H, Stein S and Lacy J: Gemcitabine plus nab-paclitaxel for advanced pancreatic cancer after first-line FOLFIRINOX: Single institution retrospective review of efficacy and toxicity. Exp Hematol Oncol 4: 29, 2015.

4. Sharma P and Allison JP: The future of immune checkpoint therapy. Science 348: 56-61, 2015.

5. Zheng L, Xue J, Jaffee EM and Habtezion A: Role of immune cells and immune-based therapies in pancreatitis and pancreatic ductal adenocarcinoma. Gastroenterology 144: 1230-1240, 2013.

6. He $\mathrm{H}$ and Baldwin GS: p21-activated kinases and gastrointestinal cancer. Biochim Biophys Acta 1833: 33-39, 2013.

7. Yeo D, He H, Patel O, Lowy AM, Baldwin GS and Nikfarjam M: FRAX597, a PAK1 inhibitor, synergistically reduces pancreatic cancer growth when combined with gemcitabine. BMC Cancer 16: 24, 2016.

8. Tyagi N, Bhardwaj A, Singh AP, McClellan S, Carter JE and Singh S: p-21 activated kinase 4 promotes proliferation and survival of pancreatic cancer cells through AKT- and ERK-dependent activation of NF- $\mathrm{BB}$ pathway. Oncotarget 5 : 8778-8789, 2014

9. Tyagi N, Marimuthu S, Bhardwaj A, Deshmukh SK, Srivastava SK, Singh AP, McClellan S, Carter JE and Singh S: p-21 activated kinase 4 (PAK4) maintains stem cell-like phenotypes in pancreatic cancer cells through activation of STAT3 signaling. Cancer Lett 370: 260-267, 2016.

10. Moon SU, Kim JW, Sung JH, Kang MH, Kim SH, Chang H, Lee JO, Kim YJ, Lee KW, Kim JH, et al: p21-activated kinase 4 (PAK4) as a predictive marker of gemcitabine sensitivity in pancreatic cancer cell lines. Cancer Res Treat 47: 501-508, 2015.

11. Biankin AV, Waddell N, Kassahn KS, Gingras MC, Muthuswamy LB, Johns AL, Miller DK, Wilson PJ, Patch AM, Wu J, et al; Australian Pancreatic Cancer Genome Initiative: Pancreatic cancer genomes reveal aberrations in axon guidance pathway genes. Nature 491: 399-405, 2012

12. Murray BW, Guo C, Piraino J, Westwick JK, Zhang C, Lamerdin J, Dagostino E, Knighton D, Loi CM, Zager M, et al: Small-molecule p21-activated kinase inhibitor PF-3758309 is a potent inhibitor of oncogenic signaling and tumor growth. Proc Natl Acad Sci USA 107: 9446-9451, 2010.

13. Huynh N, Liu KH, Baldwin GS and He H: P21-activated kinase 1 stimulates colon cancer cell growth and migration/invasion via ERK- and AKT-dependent pathways. Biochim Biophys Acta 1803: 1106-1113, 2010.
14. Yeo D, Huynh N, Beutler JA, Christophi C, Shulkes A, Baldwin GS, Nikfarjam $\mathrm{M}$ and $\mathrm{He} \mathrm{H}$ : Glaucarubinone and gemcitabine synergistically reduce pancreatic cancer growth via down-regulation of P21-activated kinases. Cancer Lett 346: 264-272, 2014.

15. Bang D, Wilson W, Ryan M, Yeh JJ and Baldwin AS: GSK-3 $\alpha$ promotes oncogenic KRAS function in pancreatic cancer via TAK1-TAB stabilization and regulation of noncanonical NF- $\mathrm{KB}$. Cancer Discov 3: 690-703, 2013.

16. Nikfarjam M, Yeo D, He H, Baldwin G, Fifis T, Costa P, Tan B, Yang E, Wen Sw and Christophi C: Comparison of two syngeneic orthotopic murine models of pancreatic adenocarcinoma. J Invest Surg 26: 352-359, 2013.

17. Chou TC and Talalay P: Quantitative analysis of dose-effect relationships: The combined effects of multiple drugs or enzyme inhibitors. Adv Enzyme Regul 22: 27-55, 1984.

18. Newman AM and Alizadeh AA: High-throughput genomic profiling of tumor-infiltrating leukocytes. Curr Opin Immunol 41: 77-84, 2016.

19. Junttila MR and de Sauvage FJ: Influence of tumour microenvironment heterogeneity on therapeutic response. Nature 501: 346-354, 2013.

20. Rooney MS, Shukla SA, Wu CJ, Getz G and Hacohen N: Molecular and genetic properties of tumors associated with local immune cytolytic activity. Cell 160: 48-61, 2015.

21. Peske JD, Woods AB and Engelhard VH: Control of CD8 T-cell infiltration into tumors by vasculature and microenvironment. Adv Cancer Res 128: 263-307, 2015.

22. Rusakiewicz S, Semeraro M, Sarabi M, Desbois M, Locher C, Mendez R, Vimond N, Concha A, Garrido F, Isambert N, et al: Immune infiltrates are prognostic factors in localized gastrointestinal stromal tumors. Cancer Res 73: 3499-3510, 2013.

23. Sideras K, Braat $\mathrm{H}$, Kwekkeboom J, van Eijck $\mathrm{CH}$, Peppelenbosch MP, Sleijfer S and Bruno M: Role of the immune system in pancreatic cancer progression and immune modulating treatment strategies. Cancer Treat Rev 40: 513-522, 2014.

24. Ino Y, Yamazaki-Itoh R, Shimada K, Iwasaki M, Kosuge T, Kanai $Y$ and Hiraoka N: Immune cell infiltration as an indicator of the immune microenvironment of pancreatic cancer. $\mathrm{Br} \mathrm{J}$ Cancer 108: 914-923, 2013.

25. Fukunaga A, Miyamoto M, Cho Y, Murakami S, Kawaradaz Y, Oshikiri T, Kato K, Kurokawa T, Suzuoki M, Nakakubo Y, et al: $\mathrm{CD}^{+}$tumor-infiltrating lymphocytes together with $\mathrm{CD}^{+}$ tumor-infiltrating lymphocytes and dendritic cells improve the prognosis of patients with pancreatic adenocarcinoma. Pancreas 28: e26-e31, 2004.

26. Hingorani SR, Wang L, Multani AS, Combs C, Deramaudt TB, Hruban RH, Rustgi AK, Chang S and Tuveson DA: Trp53R172H and KrasG12D cooperate to promote chromosomal instability and widely metastatic pancreatic ductal adenocarcinoma in mice. Cancer Cell 7: 469-483, 2005.

27. Eriksson E, Wenthe J, Irenaeus S, Loskog A and Ullenhag G: Gemcitabine reduces MDSCs, tregs and TGF $\beta-1$ while restoring the teff/treg ratio in patients with pancreatic cancer. J Transl Med 14: 282, 2016.

28. Shibuya KC, Goel VK, Xiong W, Sham JG, Pollack SM, Leahy AM, Whiting SH, Yeh MM, Yee C, Riddell SR, et al: Pancreatic ductal adenocarcinoma contains an effector and regulatory immune cell infiltrate that is altered by multimodal neoadjuvant treatment. PLoS One 9: e96565, 2014.

29. Manji GA, Olive KP, Saenger YM and Oberstein P: Current and emerging therapies in metastatic pancreatic cancer. Clin Cancer Res 23: 1670-1678, 2017.

30. Philip PA, Benedetti J, Corless CL, Wong R, O'Reilly EM, Flynn PJ, Rowland KM, Atkins JN, Mirtsching BC, Rivkin SE, et al: Phase III study comparing gemcitabine plus cetuximab versus gemcitabine in patients with advanced pancreatic adenocarcinoma: Southwest Oncology Group-directed intergroup trial S0205. J Clin Oncol 28: 3605-3610, 2010.

31. Kindler HL, Niedzwiecki D, Hollis D, Sutherland S, Schrag D, Hurwitz H, Innocenti F, Mulcahy MF, O'Reilly E, Wozniak TF, et al: Gemcitabine plus bevacizumab compared with gemcitabine plus placebo in patients with advanced pancreatic cancer: Phase III trial of the Cancer and Leukemia Group B (CALGB 80303). J Clin Oncol 28: 3617-3622, 2010. 